torting the helical structure of the phase) and the nematic phase develops when the equilibrium pitch of the cholesteric phase becomes larger than the sample thickness. This transition is first order and can easily be observed when approaching a smectic phase, because the cholesteric pitch diverges at this transition. In this way, it is possible to observe the growth of the cholesteric phase into the nematic one. The main observation is that the texture of the cholesteric phase varies with the front velocity. An example of directional growth is given in figure 3 . This transition is due to a $\pi$-rotation of the cholesteric fingers (stripes) whose ends are different due to the absence of mirror symmmetry in a cholesteric. This chirality-induced morphological transition is not yet completely understood.

In conclusion, these examples show the variety of instabilities and pattern formation that arise during the growth of liquid crystals. Most phenomena observed in liquid crystals are generic and present in other systems such as metals, alloys or polymers. In particular, the questions concerning morphological transitions, confinement effects, wavelength selection, secondary instabilies and transition to chaos or turbulent states are quite general. By contrast, problems relating to chirality are more specific but could play a role in biology-does DNA have a cholesteric texture in the cell nucleus?

The author is a research director at the Ecole Normale Supérieure in Lyon in France

\section{Further Reading}

Dynamics of Curved Fronts edited by P. Pelcé

(Academic Press, New York, 1988) - "Mesophase Growth" by J. Bechhöfer in Pattern Formation in Liquid Crystals edited by A. Buka and L. Kramer (Springer, 1996) - J.C. Géminard, P. Oswald, D. Temkin, J. Malthête Europhys. Lett. 2269 (1993) - P. Oswald, J. Bechhoefer, A. Libchaber Phys. Rev. Lett. 582318 (1987) - F. Melo, P. Oswald Phys. Rev. Lett. 641381 (1990) • J. Baudry's Thèse de I'Ecole Normale Supérieure de Lyon, 1999

Colloidal dispersions - ink, paints, lubricants, cosmetics and pharmaceuticals, and foods such as milk and mayonnaiseare - are ubiquitous in everyday life and play a key role in many industrial processes. The dispersions are essentially two-phase systems, involving mesoscopic solid or liquid particles, suspended in a liquid

\author{
Jean-Pierre Hansen, England and Peter N. Pusey, Scotland
}

\title{
Phase Behaviour of Colloidal Systems
}

$T^{\text {he sizes of colloidal particles are typ- }}$ $I$ ically in the range 10 to $10^{3}$ nanometres-they are thus much larger than atoms and molecules, but small enough that Brownian motion usually dominates gravitational settling, allowing thermodynamic equilibrium to be reached.

Solid colloidal particles (to be considered here) may be mineral crystallites, like the gold solution studied by Faraday 150 years ago, or synthetic polymeric particles, like polystyrene spheres suspended in water, or amorphous polymethylmethacrylate (PMMA) particles dispersed in hydrocarbon liquids. The impenetrable mesoscopic particles usually interact via strong, attractive, shortranged van der Waals forces, which may lead to flocculation or coagulation of the colloids into gel-like structures - this led Graham to coin their name from the Greek $\kappa o \lambda \lambda \alpha$ for "glue". Flocculation may however be prevented by either steric or electrostatic stabilization. Steric stabilization is achieved by grafting polymer "brushes" on the surface of the colloidal particles, providing an elastic repulsion when two particles come so close that their "brushes" are compressed. Colloidal particles in water generally acquire a charge by dissociation of surface groups; the charged surface and microscopic co- and counter ions in solution form electric double-layers that repel strongly whenever neighbouring surfaces get closer than the Debye screening length $\lambda_{D}$.

In their studies of Brownian motion 90 years ago, Einstein and Perrin exploited already the analogy between colloids in a liquid and atoms in a gas. There are, however, significant differences between atoms and colloids, apart from the obvious change in spatial scale. In particular, the interactions between colloidal particles may be tuned, eg by the addition of salt to a dispersion of charged colloids, which leads to a reduction of $\lambda_{D}$, or by the addition of free (non-adsorbing) polymer, which leads to an effective attraction between the colloids due to the osmotic depletion effect (explained later).

These tuneable repulsive and attractive interactions between colloidal particles lead to a rich variety of phase behaviour which has been thoroughly investigated, both experimentally and theoretically. Depending on colloid concentration, and the concentration of added ions, polymers or other species, suspensions exhibit colloidal analogues of the known phases of simple molecular systems: gas, liquid, crystalline solid and glass. Colloidal crystals in suspension are easily detected by the Bragg reflection of visible light, the wavelength of which is comparable to the colloid lattice spacing, often giving them a beautiful opalescent appearance (figures 1 and 2).

\section{Binary mixtures}

Recent research has focused on colloidcolloid mixtures and colloid-polymer mixtures. The two key parameters are the size ratio $\xi=R_{B} / R_{A}$, where $R_{A}$ and $R_{B}$ denote the radii of the two species, and the degree of non-additivity of their interactions. In simple molecular systems, $\xi$ is rarely smaller than $\sim 0.5$, while interactions are almost invariably additive. In colloidal systems, by contrast, $\xi$ can take rather extreme values (as small as 0.1 or less) and colloid-polymer interactions are highly non-additive. Thus in a mixture of two species of colloid, modelled as hard spheres, the centres of two particles cannot come closer than the sum of their radii: additive interactions. Random-coil polymer molecules, however, are soft and can interpenetrate rather easily. Nevertheless, they cannot penetrate the solid colloidal particles. Thus, in a colloidpolymer mixture, the range of the colloidpolymer interaction is greater than the sum of the ranges of the self-interactions: non-additive interactions.

The importance of non-additivity of inter-species interactions is illustrated 
strikingly by comparing the experimental phase diagrams (figures $3 \mathrm{a}$ and $3 \mathrm{~b}$ ) of a colloid-colloid mixture and a colloidpolymer mixture at almost the same size ratio, $\xi \sim 0.58$.

\section{Experiment}

Figure 3a shows the phase diagram of suspensions containing mixtures of uncharged, sterically-stabilised PMMA particles of radii $R_{A}=321 \mathrm{~nm}, R_{B}=186 \mathrm{~nm}$. Thin grafted polymer brushes ensure that the interparticle interaction is essentially that of hard spheres. The axes, $\phi_{A}$ and $\phi_{B}$, in figure $3 \mathrm{a}$ are the fractions of the sample volume occupied by each species, defined by $\phi_{A, B}=n_{A, B}(4 \pi / 3) R_{A, B}^{3}$, where $n_{A, B}$ is the number of particles per unit volume. For a total volume fraction, $\phi_{A}+\phi_{B}$, up to about 0.50 the suspension remains in a homogeneous fluid-like phase throughout which the particles diffuse in Brownian motion. For $\phi_{A}+\phi_{B}>0.50$, however, a rich variety of two- and three-phase regions of fluidsolid and solid-solid coexistence is found
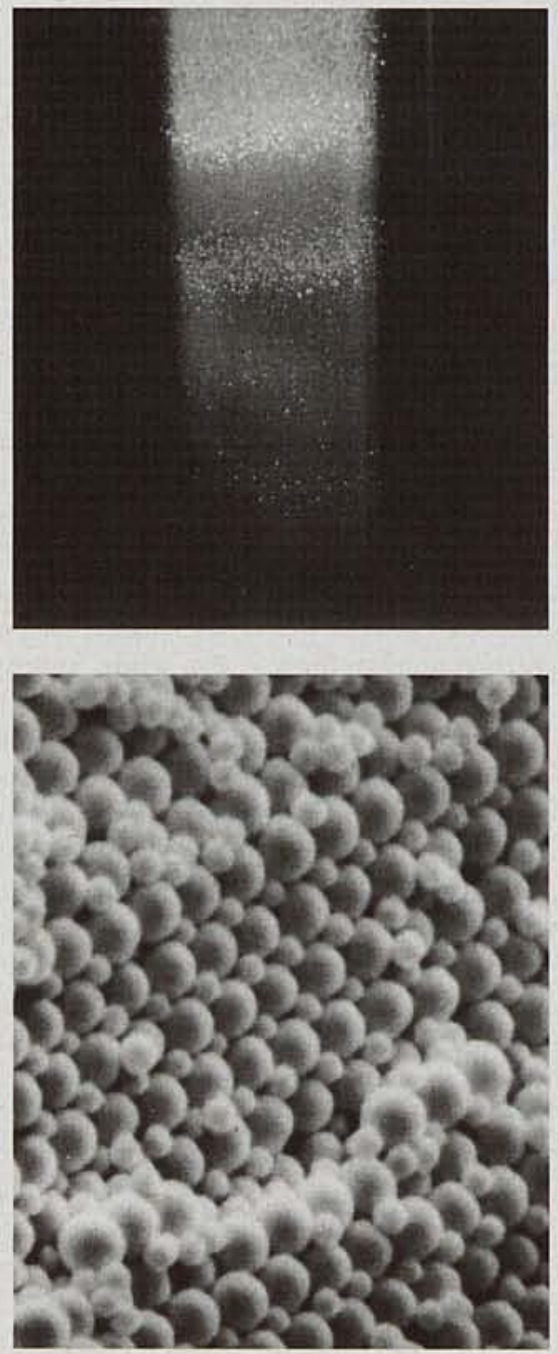

(phase separation of the initially wellmixed suspension can take several weeks). Four kinds of colloidal crystal are involved: pure $A$ and pure $B$, and the binary "colloidal alloys" $\mathrm{AB}_{2}$ and $\mathrm{AB}_{13}$.

For a one-component hard-sphere suspension, $\phi_{A}$ or $\phi_{B}=0$ (ie on one of the axes of figure 3a), colloidal crystals are first formed at $\phi_{A, B} \sim 0.50$ and crystallization is complete at $\phi_{A, B} \sim 0.55$. In the binary mixture, freezing into pure $A$ or pure $B$ is also observed near to the axes of the phase diagram. Away from the axes, however, the preferred crystalline structures are the remarkable $A B_{2}$ and $A B_{13}$ alloys. $\mathrm{AB}_{2}$ (see figure 2) is a layered structure consisting of planes of the large A particles in a triangular (or hexagonal) lattice, separated by planes containing twice as many of the smaller $\mathrm{B}$ particles. $\mathrm{AB}_{13}$ ( figure 1 ) is a yet more complex structure whose unit cell contains 112 particles. The A particles are arranged on a simple cubic lattice; oriented clusters of $13 \mathrm{~B}$ particles with an icosahedral arrangement lie

Fig 1 An $\mathrm{AB}_{13}$ colloidal crystal, comprising microscopic PMMA (polymethylmethacrylate) particles of two sizes at radius ratio $\mathrm{RB} / \mathrm{RA}=\mathbf{0 . 5 8}$. The particles are suspended in a mixture of hydrocarbon liquids chosen to nearly match the refractive index of the particles, providing a nearly transparent suspension. The sample is illuminated from behind by white light. Bragg diffraction of the light by small crystallites causes the rainbow-like scattering

Photograph by A.H. KraLL

Fig 2 Scanning electron micrograph of a dried $\mathrm{AB}_{2}$ crystal comprising submicron particles of two sizes. The crystals were formed in suspension and the liquid was then allowed to evaporate slowly over several weeks. The resulting dry "compact" of particles was fractured and sputter-coated with gold for study in the electron microscope. While some particles at the surface have been displaced, longranged binary order is clearly evident throughout the picture. Layers of large particles, viewed almost edge-on, are separated by layers of small particles

Micrograph BY A.B. SCHOFIELD within each cube.

Figure $2 \mathrm{~b}$ shows the phase diagram of suspensions containing PMMA particles of one size, radius $R_{A}=228 \mathrm{~nm}$, and random-coil polymer molecules with radius of gyration $R_{g}=130 \mathrm{~nm}$ (we take the polymer to be the $\mathrm{B}$ species so that $R_{g}=R_{B}$ ). Despite the similar size ratios, the phase behaviour of the colloid-polymer mixture (figure $3 \mathrm{~b}$ ) is markedly different from that of the colloid-colloid mixture (figure $3 \mathrm{a}$ ). Again at low concentrations, $\phi_{A}$ or $\phi_{B}<0.50$, a single fluid phase is observed. However, now at somewhat higher concentrations there is a region of coexistence of two fluid phases, dilute "colloidal gas" and concentrated "colloidal liquid", and a gas-liquid critical point. At still higher concentrations we find a threephase "triple triangle" where colloidal gas, liquid and crystal coexist (figure $3 \mathrm{~b}$ ). At the highest concentrations, gas-crystal coexistence or long-lived metastable "gels" are observed.

\section{Theory and simulation}

The theoretical description of colloidal dispersions, which are multi-component systems involving widely different length scales, requires some coarse-graining. The liquid suspension medium is generally treated as a continuum, characterized exclusively by macroscopic properties. Mixtures of hard spheres are readily simulated by Monte Carlo or Molecular Dynamics methods and, for $\xi \approx 0.58$, such simulations have led to phase diagrams involving $\mathrm{AB}_{2}$ and $\mathrm{AB}_{13}$ alloys which agree quite well with experiment (see figure $3 \mathrm{a}$ ). Intriguingly, the crystallization of hard spheres, both one- and two-component, is driven by entropic contributions to their free energies. At a high enough concentration the particles have greater freedom for local motions in the ordered structures than in the metastable fluids from which they grow: the gain in entropy associated with local "free volume" more than offsets the loss of entropy resulting from the adoption of long-ranged order.

Colloid-polymer mixtures have also been investigated by theory-in the simplest models the polymer molecules are assumed to be totally interpenetrableand by simulation, modelling the polymer by a lattice random walk. Again, reasonable agreement with experiment is found, and again the phase separations are entropically driven. The polymer molecules cannot penetrate the particles, so there is a shell, or "depletion zone", of outer radius $R_{A}+R_{g}$ around each particle from which the centres of the polymer molecules are excluded (inset, figure $3 \mathrm{~b}$ ). 


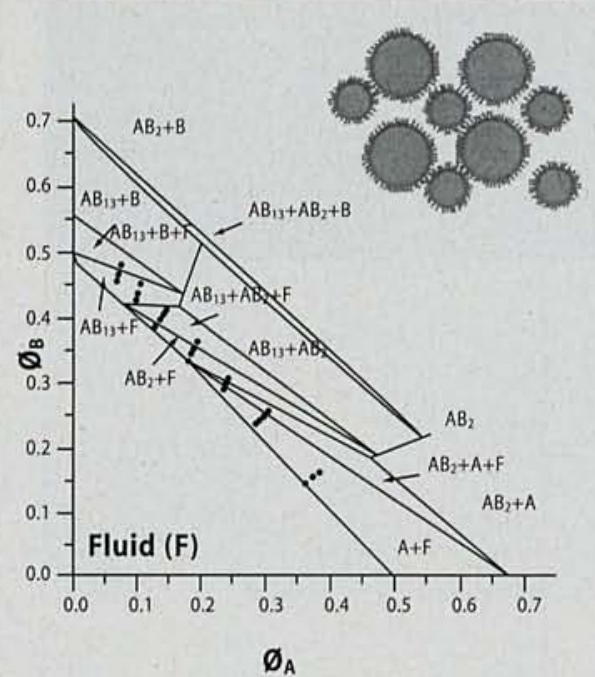

Fig 3a
Cp

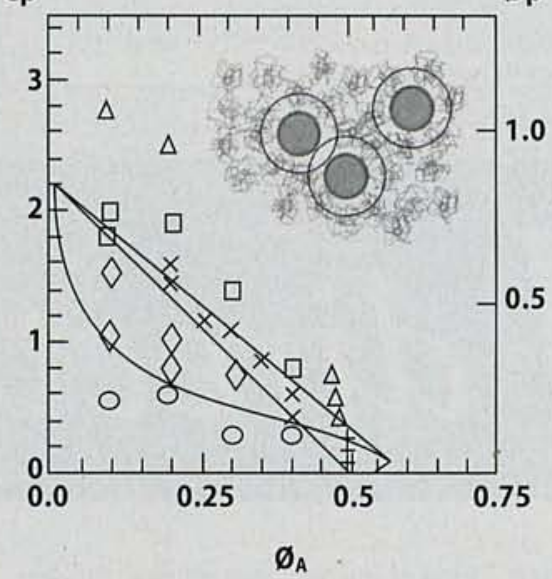

Fig 3b

Fig $3 \mathrm{~b}$ Experimental phase diagram of a mixture of colloid and non-adsorbing polymer (PMMA particles and polystyrene polymer) at polymer-to-particle size ratio $\sim 0.57$. The $x$-axis is the volume fraction of colloid; the left-hand $y$-axis gives the weight fraction of polymer $\left(c_{p}\right.$, in $\left.\mathrm{mg} \mathrm{ml}^{-1}\right)$ and the right-hand $y$-axis the polymer volume fraction $\phi_{p}$. The symbols indicate the observed phase behaviour: circles, onephase fluid; diamonds, two-phase gasliquid coexistence; plus signs, two-phase fluid-crystal coexistence; crosses, threephase gas-liquid-crystal coexistence; squares, gas-crystal coexistence; triangles, colloidal gel. Note the striking difference between Figs $3 a$ and $3 b$, reflecting the effect of non-additive colloid-polymer interactions. Inset Illustration of the depletion effect. The centres of the polymer molecules are excluded from

$\emptyset p$

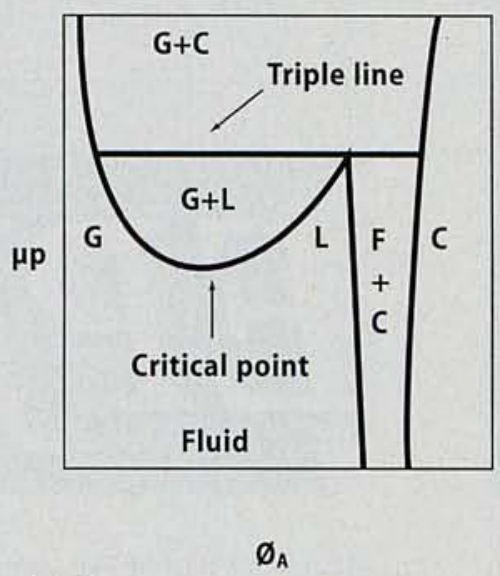

Fig $3 c$

depletion zones, indicated by the black circles, around the particles. When the depletion zones of two particles overlap, the uneven distribution of polymer around the particles results in an osmotic force pushing them together

Fig 3c Schematic of the colloid-polymer phase diagram of Fig $3 \mathrm{~b}$ replotted with the polymer chemical potential $\mu_{p}$ as the $y$-axis. Apart from being inverted, the diagram now resembles the temperature-concentration projection of the phase diagram of a simple atomic material (Gas, Liquid, Crystal).

Increasing the polymer chemical potential (by increasing its concentration) increases the strength of the interparticle depletion attraction at constant thermal kinetic energy. In an atomic material, reducing the temperature reduces the kinetic energy at constant interparticle attraction
When two particles are close enough that their depletion zones overlap, more free volume in the sample as a whole is available to the polymer, giving the suspension higher entropy. By integrating out the degrees of freedom associated with the polymer molecules, one can also usefully describe this tendency for particles to cluster in terms of an attractive "depletion force" between the particles, caused by the unbalanced osmotic pressure exerted by the polymer on their surfaces. The phase diagram of figure $3 \mathrm{~b}$ can be replotted (figure $3 \mathrm{c}$ ) so that the vertical axis is the chemical potential of the polymer, which must be the same in all coexisting phases. Now we see a phase diagram containing a gas-liquid critical point and a triple line of coexisting gas, liquid and crystal, similar (except for being upside down) to the familiar temperature-density representation of an atomic liquid.

Colloid physics is a vigorous and growing activity, exploiting close interaction between experiment, theory and simulation. There have been many exciting developments, some of them unique to colloidal systems. For example, their dynamics are slow, allowing detailed studies of metastability and the kinetics of phase transitions. New statistical mechanical approaches have been developed to describe the effects of polydispersity, the inevitable distribution of size of colloidal particles. And there is the behaviour of charged colloids, for which recent experiments and theory have raised the intrigu- ing possibility of an attraction between like charges. Finally, there is rheology, the flow behaviour of colloidal systems, a complex and technologically important subject.

The authors are professors at British universities; Jean-Pierre Hansen works at Cambridge University and Peter N. Pusey works at the University of Edinburgh

\section{Further reading}

Observation, Prediction and Simulation of Phase Iransitions in Complex Fluids edited by M. Baus, L.F. Rull and J.-P. Ryckaert (Kluwer, Dordrecht, 1995) - Binary Hard-Sphere Mixtures by M.D. Eldridge et al, Molecular Physics 84395 (1995) Colloid-Polymer Mixtures by S.M. llett et al, Physical Reiew E 511344 (1995) 